\title{
Au coatings for spaced based reflectors
}

Stefan Schwinde, Svetlana Shestaeva, Sven Stempfhuber, Henrik von Lukowicz, Jochen Kuhnhenn, et al.

Stefan Schwinde, Svetlana Shestaeva, Sven Stempfhuber, Henrik von Lukowicz, Jochen Kuhnhenn, Sven Schröder, "Au coatings for spaced based reflectors," Proc. SPIE 11852, International Conference on Space Optics ICSO 2020, 118521Q (11 June 2021); doi: 10.1117/12.2599299

SPIE Event: International Conference on Space Optics - ICSO 2021, 2021, Online Only 


\section{International Conference on Space Optics-ICSO 2020}

Virtual Conference

30 March-2 April 2021

Edited by Bruno Cugny, Zoran Sodnik, and Nikos Karafolas
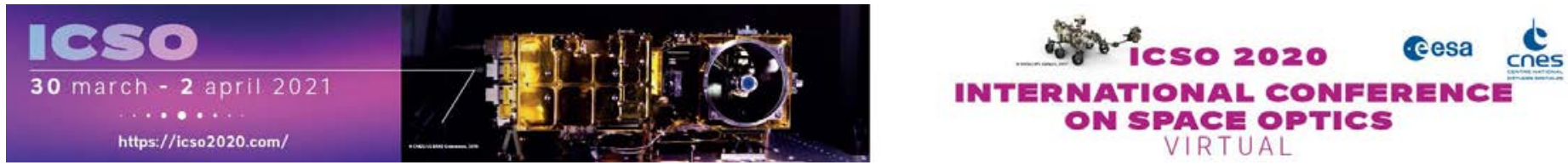

Au coatings for spaced based reflectors

\section{Cesa isoperastans ecnes}

International Conference on Space Optics - ICSO 2020, edited by Bruno Cugny, Zoran Sodnik,

Nikos Karafolas, Proc. of SPIE Vol. 11852, 118521Q · (c) 2021 ESA and CNES

CCC code: $0277-786 X / 21 / \$ 21 \cdot$ doi: $10.1117 / 12.2599299$ 


\author{
Au coatings for space based reflectors \\ Stefan Schwinde*a, Svetlana Shestaeva ${ }^{a}$, Sven Stempfhuber ${ }^{\mathrm{a}}$, Henrik von Lukowicz ${ }^{\mathrm{a}}$, Jochen \\ Kuhnhenn ${ }^{\mathrm{b}}$, and Sven Schröder ${ }^{\mathrm{a}}$ \\ ${ }^{a}$ Fraunhofer Institute for Applied Optics and Precision Engineering IOF, Albert-Einstein-Straße 7, \\ 07745 Jena; ${ }^{b}$ Fraunhofer Institute for Technological Trend Analysis INT, Appelsgarten 2, 53879 \\ Euskirchen
}

\begin{abstract}
Gold shows a very high reflectivity in the IR range. In addition, $\mathrm{Au}$ (and protected $\mathrm{Au}$ ) is more robust than $\mathrm{Ag}$ (and protected $\mathrm{Ag}$ ). Therefore, Au based coatings are of high interest. Common techniques for the deposition of optical Au coatings are sputtering and evaporation. In this contribution, both techniques, sputtering and evaporation, as well as unprotected and protected Au will be considered. A comparison of reflectivity between sputtered and evaporated Au-layers shows a slightly higher reflectivity for evaporated Au.

Beside reflectivity after coating, decrease of reflectivity due to interdiffusion at increased temperatures $\left(250^{\circ} \mathrm{C}\right)$ between adhesion layer and the reflective Au-layer is considered. In case of space-based applications, interdiffusion in thin film coatings could be activated due to particles of lower energies. This phenomenon is not necessary tested by radiation tests, performed by applying particles of higher energy. By a sputterd $\mathrm{TiO}_{\mathrm{x}}$ adhesion layer underneath a protected $\mathrm{Au}$-coating (protected by an $\mathrm{Al}_{2} \mathrm{O}_{3}-\mathrm{Si}_{3} \mathrm{~N}_{4}$-laminate), resistance against interdiffusion, and the successful passing of radiation-, cleanability- and abrasion tests could be achieved. This high reflective coating (reflectivity of $98 \%$ at $1064 \mathrm{~nm}$ ) was applied to the different mirrors of the GALA-instrument.
\end{abstract}

Keywords: Gold, coating, sputtering, reflector, interdiffusion, radiation, durability, GALA.

\title{
1. INTRODUCTION
}

Metal-based coatings show high reflectivity in a wide spectral range; therefore, they are conventionally used for many reflector applications. Aluminium (Al) has the highest reflectivity among all metals in the UV range. From $420 \mathrm{~nm}$ to the IR range, silver $(\mathrm{Ag})$ is a metal with a highest reflectivity. Beside $\mathrm{Ag}$, Gold ( $\mathrm{Au}$ ) shows a very high reflectivity in the IR range. In addition, $\mathrm{Au}$ (and protected $\mathrm{Au}$ ) is chemically more stable than $\mathrm{Ag}$ (and protected $\mathrm{Ag}$ ). Therefore, $\mathrm{Au}$ based coatings are of high interest. The James Webb Space Telescope is an impressive example for applying an Au coating to a space based mission ${ }^{1}$. Common techniques for the deposition of optical coating, based on Au, are sputtering and evaporation. In this contribution, both techniques will be considered.

In case of $\mathrm{Au}(\mathrm{Au}$ is a noble metal), the potential number of materials that can be used as adhesion layer is very limited. $\mathrm{Ti}, \mathrm{Cr}$, and the corresponding metal-oxides $\mathrm{TiO}_{2}$ and $\mathrm{Cr}_{2} \mathrm{O}_{3}$ are commonly applied as an Au-adhesion layer ${ }^{2}$. Other materials like $\mathrm{Al}$ or Indium Tin Oxide (ITO) have also been applied as an Au-adhesion layer ${ }^{2,3}$. In case of Au, interdiffusion and intermixing between Au atoms and the surroundings materials, e.g. the adhesion layer, can occur. Todeschini et al. observed a more pronounced interdiffusion for the combination " $\mathrm{Cr}$ adhesion layer beneath $\mathrm{Au}$ " than for the combination "Ti adhesion layer beneath Au"4. However, in case of contact between $\mathrm{Ti}$ and $\mathrm{Au}$, interdiffusion can also take place. Titanium tends to diffuse along the grain boundaries of Au to the surface, where it becomes oxidized ${ }^{3}$. Contact between $\mathrm{Al}$ and $\mathrm{Au}$ can lead to the formation of uniform phases like intermediate layers that consist e.g. of $\mathrm{AuAl}$ or $\mathrm{AuAl}_{2}{ }_{2}^{3,5}$. Beside these technical properties, in case of $\mathrm{Cr}_{2} \mathrm{O}_{3}$-coatings, the potential building of $\mathrm{Cr}(\mathrm{VI})$ has to be considered. $\mathrm{Cr}(\mathrm{VI}) \mathrm{can}$ cause cancers ${ }^{6}$. In this study, $\mathrm{Ti}$ and $\mathrm{TiO}_{\mathrm{X}}$ are considered as suitable adhesion layer.

Regardless of the high intrinsic chemical stability of $\mathrm{Au}$, the above described interdiffusion can lead to degradation of an $\mathrm{Au}$ coated reflector. For purpose of testing, the mentioned diffusion can be accelerated by placing samples in the surrounding with temperature higher than the operating temperatures. Regardless of this accelerated test, interdiffusion is a considerable issue for space-based applications. One of the main origins for interdiffusion can be radiation. Especially particles of lower energy can provide high levels of dose in thin film coatings ${ }^{7}$. Radiation tests are often performed by 
applying particles of higher energy, whereby the substrate is tested predominantly ${ }^{7}$. Thus, beside radiation tests, also tests at increased temperatures have been considered for a space-based application.

The GALA (Ganymede Laser Altimeter) is one of the scientific instruments of the ESA mission JUICE (Jupiter Icy Moon Explorer) with the goal of exploring the icy moons of Jupiter ${ }^{14}$. GALA is a laser altimeter that generates a surface profile and is based on an emitted laser pulse that is reflected on the surface of the investigated moon. In 2016, the Fraunhofer Institute for Applied Optics and Precision Engineering (IOF) was entrusted with the development and manufacturing of the receiving telescope. Almost all process steps from development to manufacturing, including the Au-coating, to integration and characterization have been carried out at $\mathrm{IOF}^{8}$. The manufactured mirrors (M1, M2 and tilted mirror) and the corresponding witness samples are plated with a polished NiP-layer and thus a surface roughness lower than $1 \mathrm{~nm} \mathrm{rms}$ has been achieved. For minimizing the bimetallic bending effect, a CTE matched silicon particle reinforced aluminum material with a silicon content of approx. $40 \%$ (AlSi40) is used for the mirrors and all mechanical parts ${ }^{15}$. This combination ensures a rigid, low-deformation and athermal telescope ${ }^{8}$.

$\mathrm{Au}$ is a noble metal, whereby the application of unprotected Au is feasible. However, Au is a relative soft material. A cleanable coating was required for the GALA-instrument, so only protected Au could be used. In addition, a high radiation load of electrons and protons $\left(10^{12}\right.$ protons $\left./ \mathrm{cm}^{2} @ 10 \mathrm{MeV}\right)$ was another challenge ${ }^{8}$. With regards to these challenges, the influence of adhesion layer $\left(\mathrm{Ti}\right.$ and $\mathrm{TiO}_{\mathrm{x}}$ ) on interdiffusion was considered by performing tests at increased temperatures. For passing the coating-qualification, containing an abrasion test and a radiation test, an Au based coating with a $\mathrm{Al}_{2} \mathrm{O}_{3}-\mathrm{Si}_{3} \mathrm{~N}_{4}$-laminate (protection) was applied. A successful application of the (nano-)laminates for the protection of metal-based reflectors has already been published ${ }^{9,10}$.

\section{METHODS}

\subsection{Evaporation}

The evaporated samples were prepared in a Bühler Syrus Pro 1110 evaporation chamber. The deposition of the seed layer material and the gold was carried out from one electron source, while layer thickness and deposition rate were monitored by a quartz crystal system. The evaporation material was $99.999 \%$ pure gold and $99.5 \%$ titanium.

The samples were positioned in a chamber approximately at a distance of $600 \mathrm{~mm}$ right above the electron gun. For the sample preparation, the substrates were cleaned in the chamber by plasma etching for $300 \mathrm{~s}$ and a BIAS voltage of $125 \mathrm{~V}$. At a starting pressure of $<2 \times 10^{-7} \mathrm{~Pa}$, the adhesion layer was applied to the substrate with a rate of about $0.6 \mathrm{~nm} / \mathrm{s}$. Subsequently, a $200 \mathrm{~nm}$ gold layer was deposited on the seeded substrates with a rate of about $0.2 \mathrm{~nm} / \mathrm{s}$.

For the "measurement of reflectivity of unprotected Au after coating" (see chapter 2.3) and the "exposure to increased temperatures" (see chapter 2.4) the samples E1 and E2 were manufactured by evaporation.

\subsection{Sputtering}

Sputtering was carried out in inline sputtering system with top down arrangement. The substrate scans are vertical to the narrow side of the target. The layer thickness is controlled by determining the deposition rate in a preliminary test and adjusting the number of scans and scan velocity. Preliminary to the deposition of the first layer, substrates were treated with an Ar-plasma etching in the coating chamber. Due to this procedure water and carbon residues are removed from the surface.

For the "measurement of reflectivity of unprotected Au after coating" (see chapter 2.3) and the "exposure to increased temperatures" (see chapter 2.4) sputtering were performed with an industrial direct current (DC) magnetron sputtering system MRC (Kenotec SRL, Italy). This system has three sputtering targets of $120 \times 380 \mathrm{~mm}^{2}$ and a high-frequency etching station. During deposition, the cathodes were pulsed with a DC pulse frequency of $100 \mathrm{kHz}$. Argon (Ar) was used as process gas. Ti and $\mathrm{Au}$ were sputtered using pure $\mathrm{Ti}$ or Au targets. The $\mathrm{TiO}_{\mathrm{X}}$ were sputtered reactively using $\mathrm{O}_{2}$ as reactive gas. The background pressure was below $3 \times 10^{-6}$ mbar. Sample S1 and S2 were manufactured by sputtering with this MRC system.

For the "reflectivity and durability of protected Au" (see chapter 2.6) the deposition were carried out in an inline coating system $^{11}$ that has seven targets of $100 \times 750 \mathrm{~mm}^{2}$ and a high-frequency etching station (located in two deposition chambers separated by the gate valves). Ar was used as process gas. Au, Ti and TiOx were deposited by DC magnetron sputtering. 
$\mathrm{Al}_{2} \mathrm{O}_{3}$ and $\mathrm{Si}_{3} \mathrm{~N}_{4}$ were deposited by reactive dual-magnetron sputtering with MF-sputtering (medium frequency). The oxides $\left(\mathrm{Al}_{2} \mathrm{O}_{3}, \mathrm{Si}_{3} \mathrm{~N}_{4}\right.$ and $\left.\mathrm{TiO}_{\mathrm{X}}\right)$ were sputtered reactively using $\mathrm{O}_{2}$ as a reactive gas. The background pressure was below $3 \times 10^{-6}$ mbar.

\subsection{Reflectivity of unprotected Au after coating}

An overview of the abovementioned sputtered and evaporated coatings of unprotected Au is given in the Table 1. Theses coatings were deposited on quartz glass substrates with a diameter of $25.4 \mathrm{~mm}$ and an RMS surface roughness of $\sigma_{\mathrm{RMS}} \sim 0.7 \mathrm{~nm}$ (determined by AFM in an area of $\left.10 \times 10 \mu \mathrm{m}^{2}\right)$.

Table 1. List of unprotected Au-Coatings.

\begin{tabular}{|c|c|l|}
\hline Sample ID & Deposition method & \multicolumn{1}{c|}{ Coating } \\
\hline S1 & Sputtering & Ti adhesion $+200 \mathrm{~nm} \mathrm{Au}$ \\
\hline S2 & Sputtering & TiOx-adhesion $+200 \mathrm{~nm} \mathrm{Au}$ \\
\hline E1 & Evaporation & Ti adhesion $+200 \mathrm{~nm} \mathrm{Au}$ \\
\hline E2 & Evaporation & $\mathrm{Ti}+\mathrm{TiO}_{\mathrm{x}}$-adhesion $+200 \mathrm{~nm} \mathrm{Au}$ \\
\hline
\end{tabular}

To obtain an optical performance in the wavelength range from 500 to $11000 \mathrm{~nm}$ a reflectivity of the samples was measured with spectrophotometers "Lambda 850" and "Frontier Optica FTIR" from Perkin Elmer. The performance of the coatings directly after deposition is presented in the Figure 1.

\subsection{Reflectivity of unprotected Au after exposure to increased temperatures}

Reflectivity of unprotected Au after exposure to increased temperatures was determined for the samples, which are shown in table 1. Quartz glasses with a diameter of $25.4 \mathrm{~mm}$ have been used as substrates (RMS surface roughness of $\sigma_{\mathrm{RMS}} \sim 0.7$ $\mathrm{nm}$, determined by AFM in an area of $\left.10 \times 10 \mu \mathrm{m}^{2}\right)$.

The exposure to increased temperatures was performed after evaluation of the reflectivity performance directly after deposition. Samples were exposed to $250^{\circ} \mathrm{C}$ under vacuum conditions in a SYSTEC-vacuum oven. The same sample was exposed to this test sequentially for $4 \mathrm{~h}, 24 \mathrm{~h}$ and $48 \mathrm{~h}$. Therefore, summed up total exposure time for each sample is 76 hours. After every single exposure a measurement of the coating reflectivity took place in the wavelength region from 450 to $1050 \mathrm{~nm}$. An optical performance of the samples before and after exposure to $250^{\circ} \mathrm{C}$ test-series is shown in the Figure 2 .

\subsection{Tape Test}

An adhesion between Au and adhesion layer was tested via applying a tape test according to MIL-C-48497A. With the tape 3M 853 (high tear-resistant, transparent tape of polyester) one test per sample was performed. Peeling speed: slow. Subsequently, the tested sample and tape was examined by unaided eye.

\subsection{Reflectivity and durability of protected Au}

The reflectivity and durability of protected Au is shown for substrates that are composed of an Al-alloy, with a polished NiP-layer.

Based on the findings from "reflectivity of unprotected Au after coating" and "after exposure to increased temperatures",

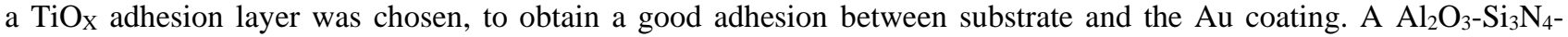
laminate was deposited as a final layer. The laminate comprises 4 pairs. Each pair consist of ca. $30 \mathrm{~nm} \mathrm{Al}_{2} \mathrm{O}_{3}$ and ca. 30 $\mathrm{nm} \mathrm{Si} \mathrm{N}_{4}$. The shown reflectivity of this coating (Fig. 3) was measured on witness samples that have been coated together with the mirrors M1, M2 or a tilted mirror respectively.

Beside the investigation of a reflectivity, a qualification has been performed on sample level. Beside other properties, the adhesion, cleanability and radiation hardness have been successfully tested. 


\section{RESULTS AND DISCUSSION}

\subsection{Reflectivity of unprotected Au after coating}

The reflectivity of the sputtered and evaporated unprotected Au is shown in Figure 1. The reflectivity in the IR is comparable for all samples. Comparison of reflectivity between sputtered and evaporated Au-layers shows a slightly higher reflectivity for evaporated Au. This difference could originate from a differing in Au-particle transport during the deposition (before arriving the substrate). In case of evaporation, the arrival of Au clusters on the substrate can lead to a beneficial layer structure ${ }^{12}$. In case of sputtering, the predominantly arrival of single atoms can be assumed.

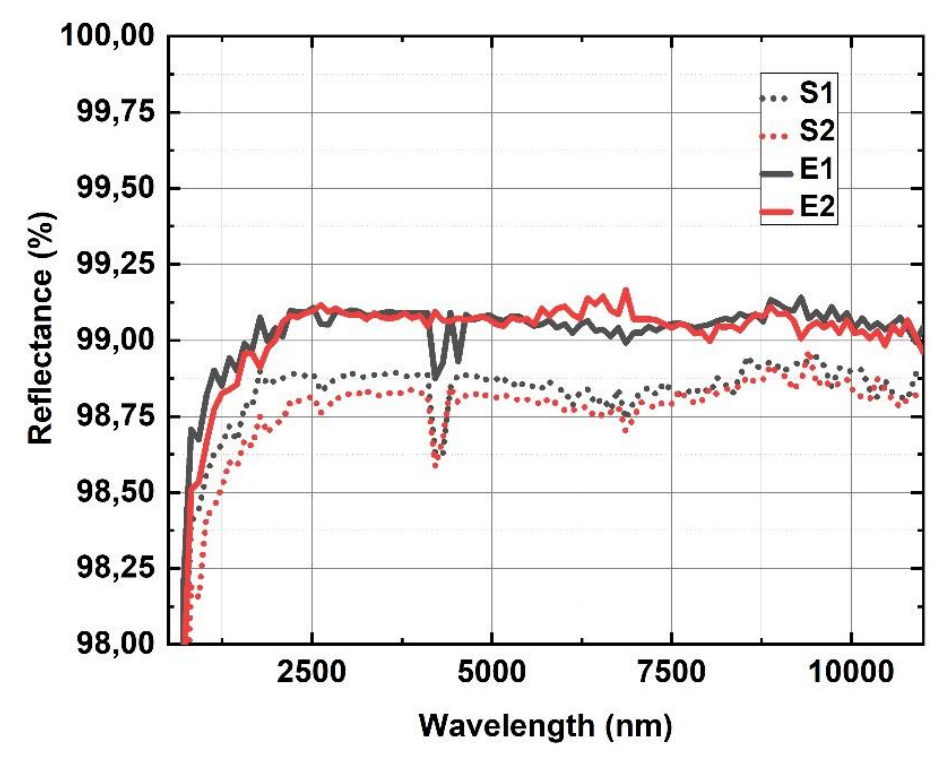

Figure 1. Reflectivity of sputtered and evaporated Au directly after deposition.

\subsection{Reflectivity of unprotected Au after exposure to increased temperatures}

Figure 2 shows the reflectivity of the unprotected $\mathrm{Au}$ (see Table 1) after coating and after exposure to $250^{\circ} \mathrm{C}$ in vacuum. The reflectivity was measured in the wavelength region from $450 \mathrm{~nm}$ to $1050 \mathrm{~nm}$, since the changes of the thin film structure are more noticeable in a shorter wavelength region.

In case of Ti underneath the Au-layer, the decrease of reflectivity is significant. At a wavelength of $750 \mathrm{~nm}$, the decrease of reflectivity is $\sim 3 \%$ for the sputtered samples $\mathrm{S} 1$ and $\sim 0,75 \%$ for the evaporated sample E1. In case of $\mathrm{TiO}_{\mathrm{X}}$ adhesion layer, a decrease of reflectivity is not notable at all for the sputtered sample S2. The evaporated sample E2 shows a slight decrease of reflectivity ( 0,5\%@ 750nm). By comparing S1 and E2, it should be noted that E2 has a Ti-layer underneath the $\mathrm{TiO}_{\mathrm{X}}$ (see Tab. 1). Under the assumption of a diffusion driven decrease of reflectivity, the coatings with $\mathrm{TiO}_{\mathrm{X}}$ adhesion layer are less prone to increased temperatures. In a case of $\mathrm{TiO}_{\mathrm{X}}$, a variety of binding energies must be considered ${ }^{13}$. However, Ti is used as a getter material for oxygen. To apply a stable compound as an adhesion layer could lead to coatings that are more stable toward (inter-) diffusion. 

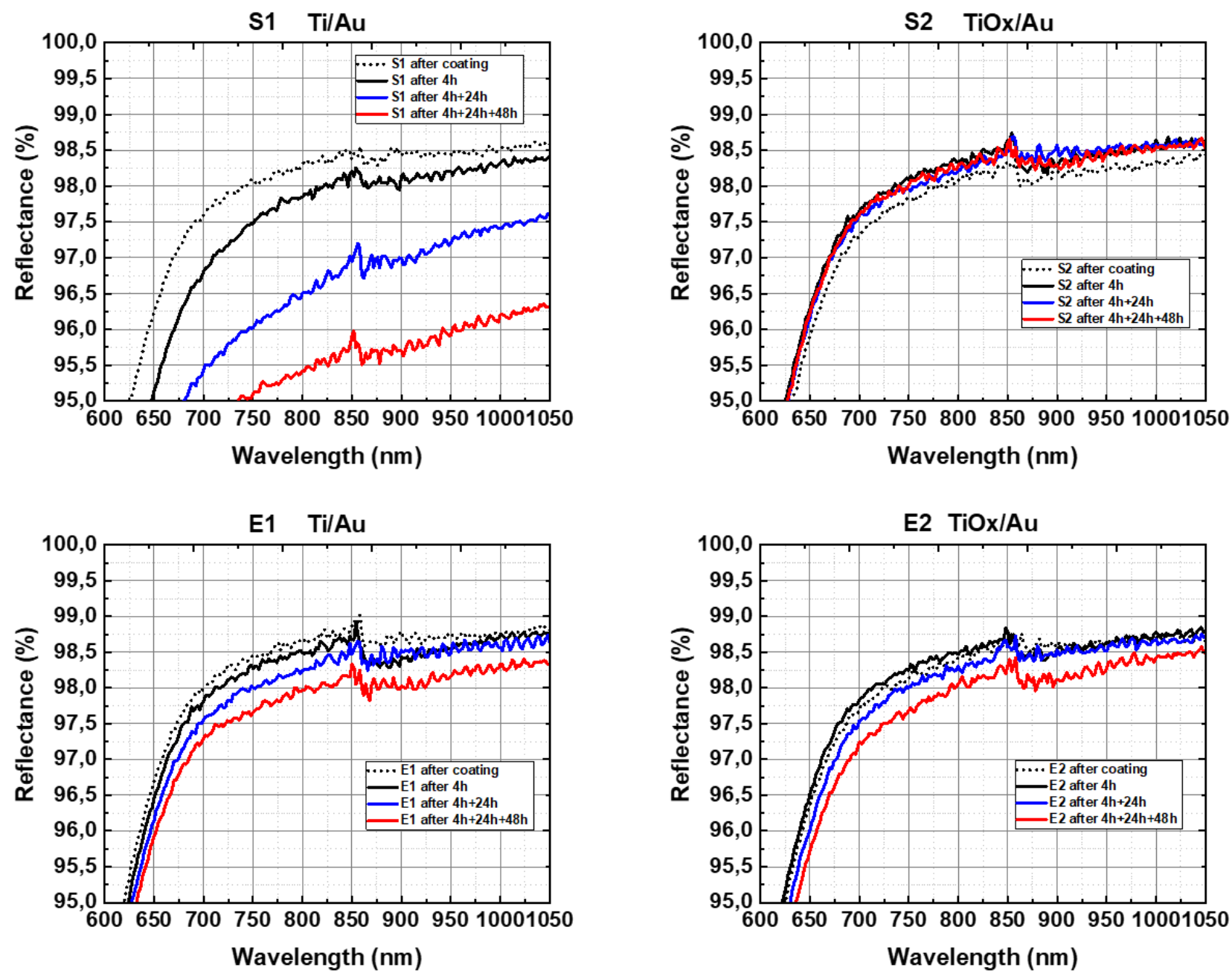

Figure 2. Decrease of reflectivity due to exposure to increased temperatures after exposure to $250^{\circ} \mathrm{C}$.

\subsection{Tape Test}

In case of the sample E2, coating was delaminated through the test. All other samples (S1, S2, E1) didn't show any degradation of the coating which can occur through this test. Since sample S2 passed the tape test, one can suggest that sputtered $\mathrm{TiO}_{\mathrm{X}}$ can be a suitable material for an Au-coatings-adhesion layer.

\subsection{Reflectivity and durability of protected Au}

The reflectivity of evaporated Au is slightly higher than the reflectivity of sputtered Au (see Figure 1). However, the sputtered coating $\mathrm{S} 2$ ( $\mathrm{TiO}_{\mathrm{X}}$ underneath $\mathrm{Au}$ ) is not prone to decrease of reflectivity due to the interdiffusion (see Figure 2). As one of the main origins for interdiffusion can be radiation, especially particles of lower energy can provide high levels of dose in coatings ${ }^{7}$, the sample $\mathrm{S} 2$ is probably more stable concerning this radiation. With regards to the adhesion, S2 passed the tape test (in contrast to E2). In addition, sputtering is suitable for the deposition of a dense protective layer. In case of sputtering, several materials can be deposited inline (without interruption of vacuum), which is certainly a further advantage for the deposition of protected and enhanced Au coatings. Therefore, sputtering technology was used for the manufacturing of a protected Au coating. An applied protective $\mathrm{Al}_{2} \mathrm{O}_{3}-\mathrm{Si}_{3} \mathrm{~N}_{4}$-laminate (see chapter 2.2), enables the cleaning of the coating. In the framework of coating qualification, an abrasion test and a cleanability test were passed. With regards to radiation hardness concerning high-energy particles, a radiation test with electrons was successfully passed. 
plication wavelength of $1064 \mathrm{~nm}$ is much larger than the single layer in a nanolaminate $(30 \mathrm{~nm})$. Thus, the laminates can be modeled as a single optical material with an effective refractive index. Taken this refractive index into account, the overall thickness of this laminate was tailored to achieve a max. reflectivity at $1064 \mathrm{~nm}$. The reflectivity at $1064 \mathrm{~nm}$ (wavelength of the GALA-application) is shown in the Figure 3. The reflectivity was measured on witness samples that have been coated together with the mirrors M1, M2 or the tilted mirror in separated batches. The very comparable curve progression (see Fig. 3) demonstrates a good reproducibility of the coating.

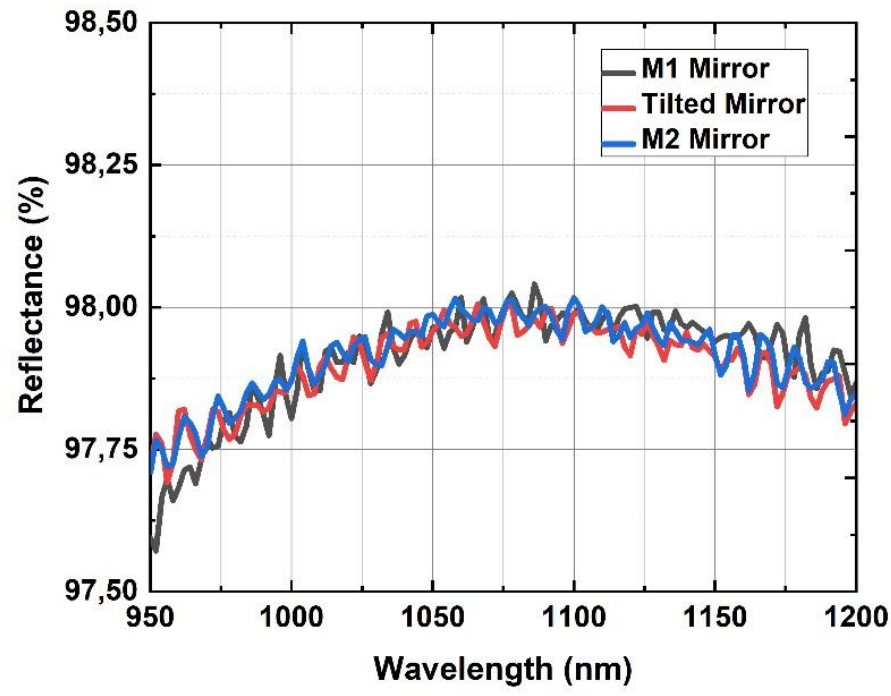

Figure 3. Reflectivity of the protected Au-coating, containing a sputtered $\mathrm{TiO}_{\mathrm{X}}$ adhesion layer and an $\mathrm{Al}_{2} \mathrm{O}_{3}-\mathrm{Si}_{3} \mathrm{~N}_{4}-\mathrm{laminate}$ as final layer.

\section{CONCLUSION}

The reflectivity of evaporated Au seems to be slightly higher than the reflectivity of sputtered Au. However, sputtered $\mathrm{Au}$ with an $\mathrm{TiO}_{\mathrm{X}}$ adhesion layer shows a good adhesion and is not prone to decrease of reflectivity due to (inter-)diffusion. The tests concerning interdiffusion have been carried out by placing samples in a surrounding with temperature, higher than the operating temperatures. Degradation due to (inter-)diffusion is also an issue for a space-based application. One of the main origins for interdiffusion in thin film coatings are particles of lower energy that can provide high levels of dose in coatings. Thus, by sputtering Au with the $\mathrm{TiO}_{\mathrm{X}}$ adhesion layer, a higher durability against this radiation can be supposed.

With regards to radiation hardness concerning high-energy particles, a radiation test with electrons was successfully passed.

In addition, sputtering is a suitable technology for the deposition of dense protective layer, like the applied $\mathrm{Al}_{2} \mathrm{O}_{3}-\mathrm{Si}_{3} \mathrm{~N}_{4-}$ laminate. In contrast to unprotected $\mathrm{Au}$, this laminate enables the successfully passing of cleanability and abrasion tests.

The overall thickness of this laminate was tailored to achieve a reflectivity of $98 \%$ at $1064 \mathrm{~nm}$. This high reflective, radiation and abrasion (cleaning) resistant coating was applied to the different mirrors of the GALA-instrument with a high reproducibility.

\section{ACKNOWLEDGEMENT}

The authors gratefully acknowledge Michael Scheler (IOF) and Thomas Müller (IOF) for the technical support and deposition of the sputtered coatings and Tim Zwerenz (IOF) for sample characterization.

Financial support for GALA was provided under grant 50 QJ 1401 on behalf of the DLR Space Administration by the German Bundesministerium für Wirtschaft und Energie. 


\section{REFERENCES}

[1] Keski-Kuha, R. A., Bowers, C. B., Quijada, M. A., "James Webb Space Telescope Optical Telescope Element Mirror Coating," Proc. of SPIE Vol. 8442 84422J, 1-12 (2012).

[2] Colas, F., Barchiesi, D., Kessentini, S., Toury, S., Lamy De La Chapelle, M., "Comparison of adhesion layers of gold on silicate glasses for SERS detection" J. Opt. 17, 114010 (2015).

[3] Poate, J. M., "Diffusion and Reactions in Gold Films", Gold Bull 14, $2-11$ (1981).

[4] Todeschini, M., Bastots da Silva Fanta, A., Jensen, F., Wagner, J. B., Han, A., „Influence of Ti and Cr Adhesion Layers on Ultrathin Au Films” ACS Appl. Mater. Interfaces 9, 37374-37385 (2017).

[5] Sritharan, T., Li, Y. B., Xu, C., Zhang, S., „Oxidation of Al-Au intermetallics and its consequences studied by X-ray photoelectron spectroscopy", J. Mater. Res. 23, (2008).

[6] Deng, Y., Wang M., Tian T. et al., "The Effect of Hexavalent Chromium on the Incidence and Mortality of Human Cancers: A Meta-Analysis Based on Published Epidemiological Cohort Studies," Frontiers in Oncology, Volume 9, Article 24 (2019).

[7] Kuhnhenn, J., Steffens, M., Baum, M., „Radiation Testing of Optical Coatings - Better Testing with Simulations”, Proc. Of SPIE Vol. 11180, 111804L-2 (2018).

[8] Von Lukowicz, H., Risse, S., Müller, S., et al., "Development of the telescope of the GALA Ganymede Laser Altimeter," to be published in annual-report of the Fraunhofer IOF 2020 (2021).

[9] Schürmann, M., Schwinde, S., Kaiser, N., OPTICAL ELEMENT COMPRISING A REFLECTIVE COATING, WO 2015/193195 AI

[10] Schwinde, S., Schürmann, M., Schlegel, R., „Protected silver coatings for reflectors, “ CEAS Space Journal, Volume 11, Issue 4, 579-587 (2019).

[11] Schürmann, M., Jobst, P.J., Yulin, S., et al. "Optical reflector coatings for astronomical applications from EUV to IR,“ Proc. SPIE 8450, 84502K (2012).

[12] Švorčik, V., Slepička, P., Švorčikova, J. et al., "Characterization of Evaporated and Sputtered Thin Au Layers on Poly(ethylene terephtalate)," Journal of Applied Polymer Science, Vol. 99, 1698-1704 (2006).

[13] Atuchin, V., V., Kesler, V., G., Pervukhina, N. V., Zhang, Z., "Ti 2p and O 1s core levels and chemical bonding in titanium-bearing oxides", Journal of Electron Spectroscopy and Related Phenomena 152, 18-24 (2006).

[14] Hussmann, H., Lingenauber, K., Kallenbach. R., et al., "The Ganymede laser altimeter (GALA): key objectives, instrument design, and performance", CEAS Space Journal 11, 381-390 (2019).

[15] Kinast, J., Hilpert, E., Lange, N., Gebhardt, A., Rohloff, R. R., et al. „Minimizing the bimetallic bending for cryogenic metal optics based on electroless nickel”, Proc. of SPIE Vol. 915136, 1-9 (2014). 